\title{
Low-wavenumber Raman scattering in glycerol
}

\author{
C. Moura† and A. Zwick*
}

Laboratoire Physique des Solides, Université Paul Sabatier, 118 route de Narbonne, 31062 Toulouse Cédex-4, France

Received 1 August 2000; Accepted 15 October 2000

\begin{abstract}
Analysis of the low-wavenumber Raman data of glycerol as a function of temperature is presented. For temperatures lower than the glass transition temperature the scattering intensity is interpreted on the basis of a model starting from the analogies between the density of vibrational states for the crystalline and the glassy phases and taking into account multiple-order scattering. Experimental results on depolarized Raman spectra are shown to be in good agreement with the model. For temperatures higher than the glass transition temperature, the quasi-elastic scattering is interpreted in terms of degrees of freedom released by H-bond breaking. Copyright $\odot 2001$ John Wiley \& Sons, Ltd.
\end{abstract}

\section{INTRODUCTION}

The low-wavenumber Raman spectra of glasses present two features that are not completely understood. First, at low temperature, the so-called boson peak (BP), not observed in crystals, is characteristic of the solid phase and persists even at temperatures higher than the melting-point. Second, quasielastic scattering (QS), characteristic of the liquid phase, is observed with intensity that is weak below the glass transition temperature $\left(T_{\mathrm{g}}\right)$, but increases with temperature faster than expected from the Bose population factor.

In this work we found that in solid glycerol, extensively studied in recent years, ${ }^{1}$ first- and second-order scattering account well for the shape of the low-wavenumber Raman spectrum. For temperatures higher than $T_{\mathrm{g}}$ an Arrhenius approach highlights the role played by H-bonds in QS.

\section{EXPERIMENTAL AND RESULTS}

Raman measurements in glycerol (Aldrich) were obtained on a Jobin Yvon T64000 spectrometer with a triple monochromator single-channel setup. The output signals were detected by standard photon counting techniques. The light sources were 488.0 and $514.5 \mathrm{~nm}$ radiation from an argon ion laser operating at a power level of about $100 \mathrm{~mW}$ at the sample. Both sets of measurements were performed using an Oxford Instruments helium cryostat with right-angle geometry, keeping the polarization of the scattered light vertical and the polarization of the incoming beam horizontal.

Figure 1 shows the depolarized Stokes and antiStokes spectra in the temperature range 93-310 K, after

*Correspondence to: A. Zwick, Laboratoire Physique des Solides, Université Paul Sabatier, 118 Route de Narbonne, 31062 Toulouse Cédex-4, France; e-mail: zwicka@ramansco.ups-tlse.fr ${ }^{\dagger}$ Permanent address: Dept. de Física, Universidade do Minho, Largo do Paço, 4719 Braga Codex, Portugal. photomultiplier (PM) noise subtraction and corrections for the apparatus response. The sample temperature was determined from the ratio between Stokes and anti-Stokes intensities. The low-wavenumber $\left(<150 \mathrm{~cm}^{-1}\right)$ Raman spectra present the well known features of vitreous materials: BP (at low temperature) and QS (at higher temperature). Figure 1 shows that the high-wavenumber modes $\left(400-600 \mathrm{~cm}^{-1}\right)$, corresponding to intramolecular vibrations, change little with temperature. One observes, as expected, a shift and a broadening of these bands. It is also clear from Fig. 1 that with increasing temperature the QS becomes very intense above $T_{\mathrm{g}}$. At the same time the maximum wavenumber of the BP scattering slightly shifts to lower wavenumbers and almost merges into QS at room temperature. Such a behaviour is general for all vitreous substances and is attributed to the co-existence of at least two kinds of excitations. However, the relative contributions due to the orientational relaxation or to the softening of the vibrations have not yet been clearly determined.

\section{Solid phase}

For temperatures far below $T_{\mathrm{g}}$, relaxational contributions can be neglected, ${ }^{2}$ and so the observed Raman scattering is due only to vibrational processes. At these temperatures the Raman spectra are generally analysed in terms of activation of the density of vibrational states (DVS), given by ${ }^{3}$

$$
I(\omega, T)=[n(\omega, T)+1] \frac{C(\omega)}{\omega} g(\omega)
$$

where $I(\omega, T)$ is the first-order Raman scattering, $g(\omega)$ is DVS, $C(\omega)$ the light-to-vibration coupling constant and $n(\omega, T)$ the Bose population factor.

It has been shown that multiple-order effects should be invoked to explain the Raman intensity, for noncrystalline materials, in the low-wavenumber range. ${ }^{4}$ The total Raman scattering is obtained as the sum of the first-order 


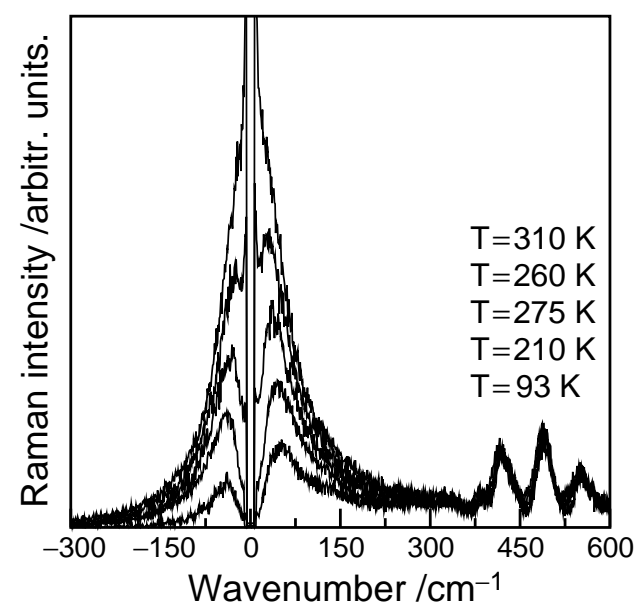

Figure 1. Depolarized Raman spectra of glycerol as function of temperatures (temperature decreasing from top to bottom).

Raman scattering, estimated by Eqn (1), and second-order contributions, ${ }^{5}$ obtained by the autoconvolution of the firstorder intensity. ${ }^{4,5,10}$ Calculation of the Raman spectrum needs knowledge of the coupling coefficient $C(\omega)$, of the DVS $g(\omega)$ and of the factor accounting for the decrease in the efficiency.

Comparison of Raman, neutron scattering and heat capacity data ${ }^{2,6,7}$ shows that the coupling coefficient $C(\omega)$ has a linear dependence in the BP region.

The DVS can be obtained by simulations methods or experimentally by inelastic neutron scattering (INS). The low-wavenumber DVS for glassy glycerol obtained from neutron data $^{8}$ at $100 \mathrm{~K}$ is presented in Fig. 2(a). Since the

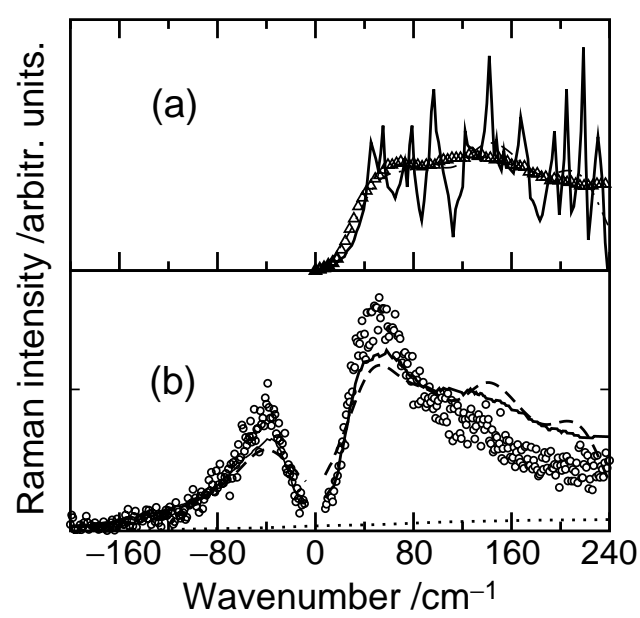

Figure 2. (a) Density of vibrational states: crystal (line); glass, smoothed (long dashed line); experimental (triangles).

(b) Calculated Raman intensity resulting from the model: smoothed crystal DVS (full line) and experimental neutron DVS (dashed line) compared with experimental depolarized spectrum (open circles). Second-order contributions (dotted line). short-range order is the same in the crystalline and glassy phases, the dynamic properties will be also similar. ${ }^{4}$ Hence the DVS of vitreous glycerol should only differ from that of the corresponding crystal by some redistribution in energy of the vibrational states. This DVS is obtained by convoluting the crystal DVS 9 [also reported in Fig. 2(a)] with a Gaussian function. In these DVSs the number of states between 0 and $200 \mathrm{~cm}^{-1}$ is the same. Comparison between the two glassy DVSs shows that the calculated value provides a good representation of the overall experimental wavenumber distribution.

In Fig. 2(b) is depicted the experimental depolarized Raman spectrum for $T=93 \mathrm{~K}$ and the calculated Raman spectra obtained using the two DVS of Fig. 2(a). The integrated intensities between -200 and $200 \mathrm{~cm}^{-1}$ in the calculated spectra are approximately the same, and the experimental spectrum is normalized to its mean value. Also represented are the contributions of the second-order processes. Being practically the same in the two cases, their relative intensity is taken to be about $10 \%$ as in all the other systems. ${ }^{5,10}$ This contribution, generally neglected at low temperatures, must be taken into account in the evolution of the low-wavenumber intensity when raising the temperature.

The experimental intensity and the maximum wavenumber of the BP are very well represented by the calculated spectra. Also very well represented is the QS, attributed, at this temperature, to second-order processes. Multiple-order contributions being almost energy independent, the remaining discrepancies between the calculated and experimental spectra are due either to some mistakes in the estimation of the DVS or to small deviations in the proportionality to $\omega$ of the coupling constant. In fact, the observed proportionality to $\omega$ of $C(\omega)$ is due to the superposition of propagative sound-like waves whose coupling factor is proportional to $\omega^{2}$ and localized extra states and optical or zone-edge acoustic phonon-like states exhibiting a specific Raman activity.

Despite these restrictions, we conclude that the major contributions to BP are associated with vibrational crystallike states.

\section{Liquid phase}

Increasing temperature leads to an increase in the central contribution in the Raman spectra, extending up to $100 \mathrm{~cm}^{-1}$. At a given wavenumber the intensity grows faster than expected from the Bose population factor only. This central contribution can be obtained by subtracting from the total spectrum the low-temperature contribution restored to high temperature, using the following expression:

$$
\Delta I=I(T)-\frac{I\left(T_{0}\right)}{n\left(\omega, T_{0}\right)+1}[n(\omega, T)+1]
$$

The quantity $\Delta I$ represents the excess of intensity due to relaxational processes or multiple-order vibrational processes. The former is related to rotations, reorientations or 


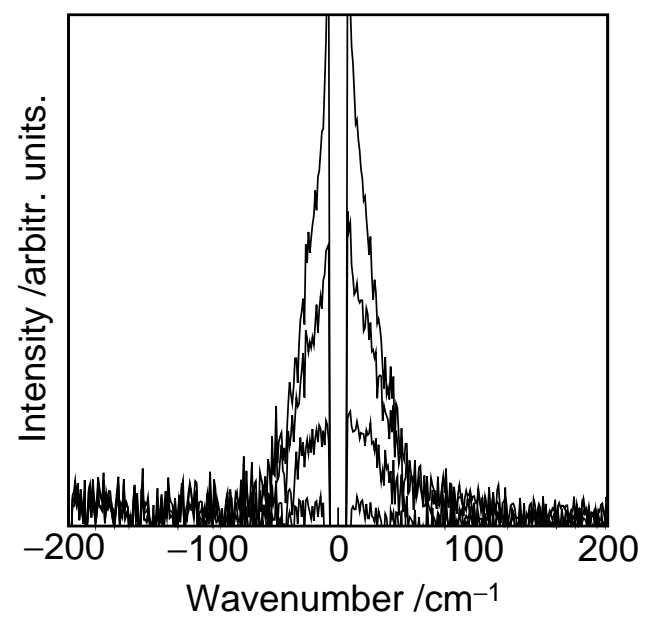

Figure 3. Quasi-elastic depolarized scattering intensity from $T=93 \mathrm{~K}$ (bottom) to $310 \mathrm{~K}$ (top).

collisions. The intensity excess is represented in Fig. 3 from 93 to $310 \mathrm{~K}$. Its behaviour can be understood as follows: in the glassy state the molecular units present in the system are frozen in the network and the contributions to the scattering are due only to vibrational processes, since reorientational or collisional processes are not present. Increasing the temperature up to fusion leads to the breaking of $\mathrm{H}$-bonds and consequently new degrees of freedom are released, giving rise to new modes participating in the central diffusion. In the studied temperature range collisional process can be neglected and taking the same contribution to the QS for each mode, the total intensity can be evaluated by an Arrhenius approach.

In Fig. 4 we present the evolution with temperature of the integrated intensity excess, between -150 and $150 \mathrm{~cm}^{-1}$ (except the contribution between -8 and $8 \mathrm{~cm}^{-1}$ ). As expected, an increase in the integrated intensity excess takes place near $T_{\mathrm{g}}$ (shown by an arrow). Also represented is the fit of the integrated intensity, giving an activation energy of $4 \mathrm{kcal} \mathrm{mol}^{-1}(1 \mathrm{kcal}=4.184 \mathrm{~kJ})$. This value, close to the hydrogen bond energy in alcohols, ${ }^{11}$ indicates that the breaking of these bonds provides the major contribution to the central intensity.

\section{CONCLUSION}

We have shown that the depolarized Raman spectrum of vitreous glycerol could be described on the basis of a model based on the redistribution of some vibrational

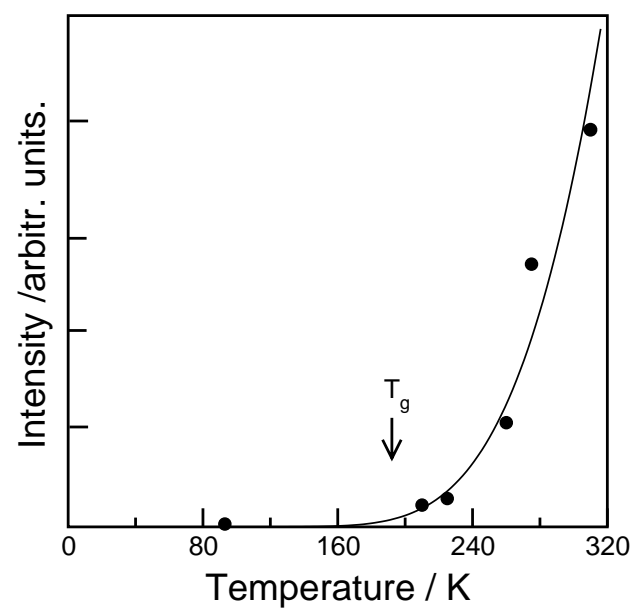

Figure 4. Total quasi-elastic depolarized scattering intensity as a function of temperature. Arrhenius approach (solid line).

states and multiple-order effects. The close correspondence between the experimental and calculated spectra proves that in the investigated wavenumber range most of the vibrational states are closely related to phonons of the crystal.

For $T<T_{\mathrm{g}}$ the central intensity is essentially due to multiple-order processes. For $T>T_{\mathrm{g}}$ the relaxational processes are associated with breaking of hydrogen bonds.

\section{REFERENCES}

1. Wuttke JJ, Hernandez J, Li G, Coddens G, Cummins HZ, Fujara F, Petry W, Sillescu H. Phys. Rev. Lett. 1994; 72: 3052, and references cited therein.

2. Achibat T, Boukenter A, Duval E. J. Chem. Phys. 1993; 99: 2046.

3. Shuker R, Gammon RW. Phys. Rev. Lett. 1970; 25: 222.

4. Carles R, Zwick A, Moura C, Djafari-Rouhani M. Philos. Mag. B 1998; 77: 397, and references cited therein.

5. Zwick A, Carles R. Phys. Rev. B 1993; 48: 6024.

6. Sokolov AP, Kisliuk A, Quitmann D, Duval E. Phys. Rev. B 1993; 48: 7692.

7. Malinovsky K, Novikov VN, Parshin PP, Sokolov AP, Zemlyanov MG. Europhys. Lett. 1990; 11: 43.

8. Cuello GJ, Bermejo FJ, Fayos R, Fernández-Perea R, Criado A, Trouw F, Tam C, Schober H, Enciso E, Almarza NG. Phys. Rev. B 1998; 57: 8254.

9. Bermejo FJ, Criado A, de Andres A, Enciso E, Schober H. Phys. Rev. B 1996; 53: 5259.

10. Chehaidar A, Zwick A, Carles R, Bandet J. Phys. Rev. B 1994; 50: 5345.

11. Luck WA. In Structure of Water and Aqueous Solution, Proceedings of the International Symposium, Luck WAP (ed.). Verlag Chemie: Weinheim, 1973; 247. 